\title{
The distribution and direction of extreme geomagnetic fluctuations over 1-60 minute periods
}

\author{
Neil C. Rogers* (1), James A. Wild ${ }^{(1)}$, and Emma F. Eastoe ${ }^{(2)}$ \\ (1) Space and Planetary Physics, Lancaster University, Lancaster, UK. https://www.lancaster.ac.uk \\ (2) Dept. Mathematics and Statistics, Lancaster University, Lancaster, UK. https://www.lancaster.ac.uk
}

\begin{abstract}
We present a statistical climatological study of extreme fluctuations in the horizontal geomagnetic field $(d B / d t)$, generated at the Earth's surface by extreme electrical currents in the ionosphere and magnetosphere. Applying techniques from Extreme Value Theory we predict the magnitude of $|d B / d t|$ for return periods (RP) from 5 to 500 years. Results presented for both ramp changes and RMS variations over periods from 1-60 minutes show considerable variation in magnitude and rate of occurrence when considered as functions of magnetic latitude, local time, and season. The direction of extreme geomagnetic fluctuations is strongly isotropic at lower latitudes, but this changes with timescale. A technique for improving return level estimates by combining Generalised Pareto tail distributions from discrete directional sectors produces return levels that are higher at low geomagnetic latitudes but higher at mid to high latitudes.
\end{abstract}

\section{Introduction}

Modelling the risk of damaging Geomagnetically Induced Currents (GICs) in ground-based infrastructure requires a statistical characterisation of the geoelectric field, $\boldsymbol{E}$, induced by electrical currents in the ionosphere and magnetosphere. This information could, for example, be combined with a model of electrical impedances in a highvoltage (HV) electricity network to determine the 'return level' of GIC expected in a 'return period' of 100 years or more. Since direct measurements of $\boldsymbol{E}$ are not globally extensive and do not cover the decades required for accurate prediction over long return periods, we have instead used a global archive of measurements of the rate of change of the horizontal component of the geomagnetic field, $\boldsymbol{d} \boldsymbol{B} / \boldsymbol{d} \boldsymbol{t}$. Using magneto-telluric theory [1] these may be combined with a model of the local ground conductivity to determine climatological statistics for $\boldsymbol{E}$.

Most previous statistical studies have examined only the 1minute changes in $\boldsymbol{B}$, often with little regard to the compass direction, $D$, of such fluctuations. However, the damaging impacts of GIC will depend on the direction of $\boldsymbol{E}$ relative to the principal axis of any element of cable, or pipeline, etc. (To a good approximation, $D$ is $90^{\circ}$ anticlockwise from the ionospheric electrical current direction, as viewed from above.) The oscillation frequency of the geomagnetic disturbance affects the calculation of $\boldsymbol{E}$ since the ground conductivity tensor is frequency-dependent. The duration of an extreme geomagnetic disturbance also determines its impact on systems: For example, HV transformer core failure due to heating is more likely after several minutes of sustained geomagnetic disturbance [2].

In this paper we extend our global climatological statistical model of 1-minute fluctuations [3] to include the magnitude and frequency of occurrence of extreme $|d B / d t|$, over periods at $1,10,30$ and 60 minutes, both as ramp changes and as a root-mean-square (RMS) variations. This range of periods is important for GIC modelling but different ionospheric and magnetospheric processes will dominate at each end of the range. Extreme value theory was used to estimate return levels for return periods from 5 to 500 years. Occurrence rates and return levels (RL) were found to depend strongly on corrected geomagnetic (CG) latitude, magnetic local time (MLT), and season, and may be anisotropic in their direction. We present a small selection of these statistics in this paper. Finally, we present a technique derived from extreme ocean wave height analysis [4] that has the potential to improve RL estimates where occurrences are strongly anisotropic in direction.

\section{Measurements and Data Processing}

125 magnetometers in the global SuperMAG collaboration [5] provided measurements of the north and east components of the magnetic field, $\left(B_{N}, B_{E}\right)$ at 1-minute cadence. There was an average of 28 years' and a minimum of 20 years' data per site. From these measurements we determined both the absolute rate of change, $|d B / d t|$, and the compass direction of the fluctuations. Details of magnetometer locations and the data processing and quality checking are given in [3]. We define $|d B / d t|$ as

$$
\left|\frac{d B}{d t}\right|=\frac{1}{\Delta t} \sqrt{\left(\Delta B_{N}\right)^{2}+\left(\Delta B_{E}\right)^{2}}
$$

where $\Delta t=1$ minute, $\Delta B_{N}(j)=B_{N}(j)-B_{N}(j-1)$ and similarly for $\Delta B_{E}(j)$. Over an n-minute period, the Ramp change in $\boldsymbol{B}$ was defined as

$$
\begin{aligned}
& \mathrm{R}_{\mathrm{n}}(\mathrm{i}) \\
& =\frac{1}{\mathrm{n} \Delta t} \sqrt{\left(B_{N}(\mathrm{i})-B_{N}(\mathrm{i}-\mathrm{n})\right)^{2}+\left(B_{E}(\mathrm{i})-B_{E}(\mathrm{i}-\mathrm{n})\right)^{2}}
\end{aligned}
$$


In practice this was implemented using moving average filters on $\Delta B_{N}$ and $\Delta B_{E}$. RMS variations over an n-minute period were also calculated as

$$
\mathrm{S}_{\mathrm{n}}(\mathrm{i})=\sqrt{\frac{1}{n} \sum_{j=i-n+1}^{i}\left|\frac{d B_{H}}{d t}\right|(j)^{2}}
$$

and this was applied using a convolution filter.

Since we are only interested in extreme values, a high threshold for $|d B / d t|$ was set at the $99.97^{\text {th }}$ percentile, $P_{99.97}$, at each magnetometer for each metric (Eqn 1-3). To ensure temporal independence, threshold exceedances were declustered to ensure a minimum 12 hours between clusters and only the peak value in each cluster was recorded. Declustered exceedances were then fitted to a Generalised Pareto (GP) probability 'tail' distribution and the fitted GP profile was used to predict return levels (RL) expected over return periods (RP) of up to 500 years (see [1] and [6] for details).

\section{Results}

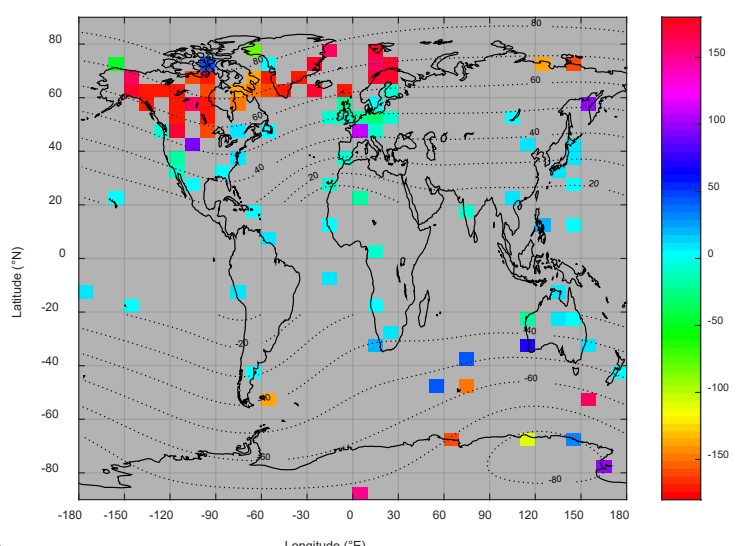

a)

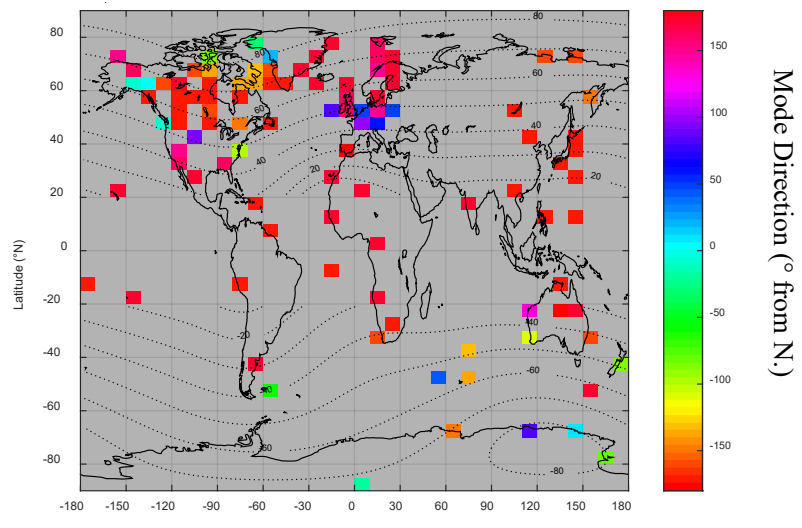

b)

Figure 1. Mode values of $D$ (in $10^{\circ}$-wide bins, centred at $0^{\circ}, 10^{\circ}$, etc.) for cluster peaks above $P_{99.97}$. a) 1 -minute fluctuations, b) 60-minute ramp change. Contours indicate corrected geomagnetic latitudes (IGRF Epoch 2000).

Figure 1 presents the mode of the direction, $D$, of each cluster peak for a) 1-minute fluctuations, and b) 60-minute ramp changes, $R_{60}$. For low geomagnetic latitudes $\left(\lambda<40^{\circ}\right)$ the 1 -minute fluctuations are principally north directed, whereas ramp changes over 60 -minute periods are south directed. The short-lived northward changes are largely identified as storm sudden commencements or sudden impulses, the result of shocks in the solar wind which temporarily enhance eastward Chapman-Ferarro currents flowing along the dayside magnetopause boundary. The southward variations over 60-minutes are largely associated with westward ring current enhancements during the Main Phase of geomagnetic storms.

Maximum likelihood estimates (MLE) of the 100-year return levels for the 1-minute $|d B / d t|$ at each of the 125 magnetometer sites, are shown as magenta ' + ' in Figure 2, where error bars indicate $95 \%$ confidence intervals (CI).

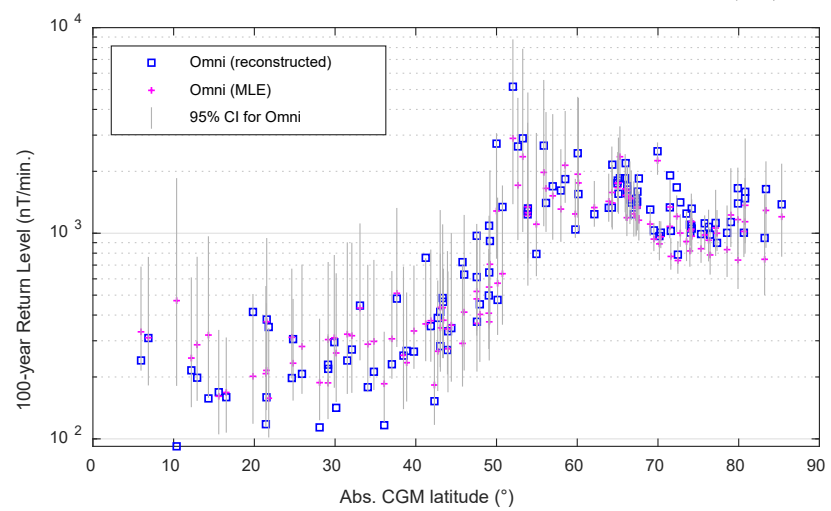

Figure 2. 100-year RL predictions vs CGM latitude comparing results reconstructed from eight $45^{\circ}$ directional bins (blue), with those ignoring direction (magenta).

The sharp peak in RL up to approximately $3000 \mathrm{nT} / \mathrm{min}$ at CG latitude, $|\lambda|=53^{\circ}$, was observed in both hemispheres. These may be associated with intense auroral substorm expansions - intensifications of westward ionospheric currents in auroral arcs near the equatorward edge of a greatly expanded auroral electrojet region. A secondary increase in RLs towards the geomagnetic poles is observed for return periods of 100 years or more, associated with long-period oscillations during the summer months around noon for northward interplanetary magnetic field conditions, when the strong geomagnetic dipole tilt is favourable to magnetic reconnection in the magnetospheric tail lobe [3].

Figure 3 maps $\operatorname{Pr}\left(|d B / d t|>P_{99.97}\right)$ against $C G$ latitude and MLT for a) 1-minute fluctuations (Eqn. 1), b) 60-minute ramp changes, $R_{60}$ (Eqn. 2), and c) 60-minute RMS variations, $S_{60}$ (Eqn. 3). For 1-minute fluctuations, at auroral latitudes $\left(65^{\circ}<|\lambda|<75^{\circ}\right)$ the $\operatorname{Pr}\left(|d B / d t|>P_{99.97}\right)$ peaks in the hours before local midnight and their directions (not shown) are predominantly southward. Occurrence rates are also strongly dependent on month of the year (not shown), and influenced by the orientation of the interplanetary magnetic field (IMF).

It is interesting to observe that the greatest occurrence of extreme 60-minute RMS changes occurs in the dawn sector 
(04-07 MLT) in the auroral zone (Figure 2c). This region is characterised by strong and sustained ULF wave activity (Pc5 pulsations) as discussed in [3].

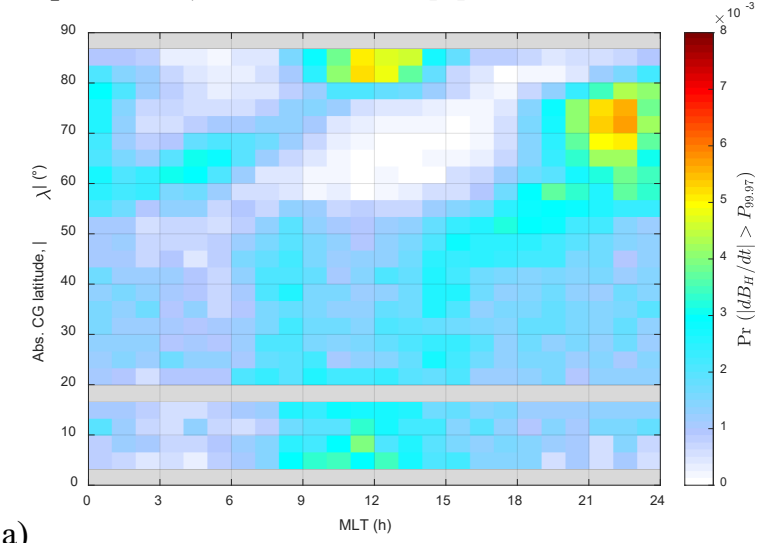

a)

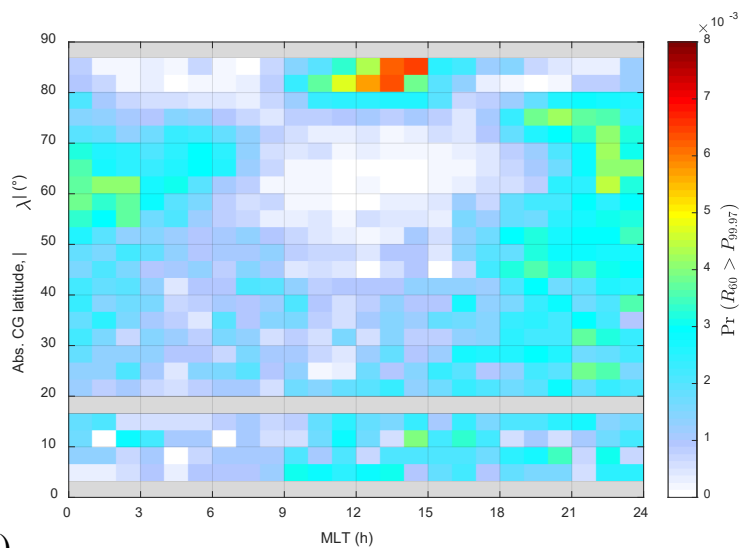

b)



c)

Figure 3. Occurrence probability of cluster peaks. a) 1minute fluctuations (Ramp or RMS), b) 60-minute Ramp change, c) 60-minute RMS variation.

The strong dependence of $P\left(|d B / d t|>P_{99.97}\right)$ on covariates (direction, MLT, month, etc.) violates the assumption of an identical distribution in fitting the GP distribution and inferring RLs. It has been postulated that models that take into account covariate effects may yield more accurate results since they explain more of the variability in the data by setting thresholds that vary with the covariate. The following example - based on an ocean wave height analysis method [4] - applies to peaks of $|d B / d t|$ in discrete directional sectors, but can easily be adapted for seasonal and local time sectors.
Taking $k$ non-intersecting $45^{\circ}$-wide directional sectors we determined a sufficiently high threshold, $u_{i}$ in each sector, $i$. (We initially tried $u_{i}=P_{99.97}$ for each sector.) In each sector, we fitted parameters of the complementary GP distribution of $\boldsymbol{x}=\left|d B_{H} / d t\right|$ conditional on exceeding $u_{i}$, given by

$$
\operatorname{Pr}\left(X_{i}>\boldsymbol{x} \mid X_{i}>u_{i}\right)=\left[1+\frac{\xi_{i}\left(\boldsymbol{x}-u_{i}\right)}{\sigma_{i}}\right]_{+}^{-1 / \xi_{i}}
$$

where $X_{i}$ denotes a measurement in sector $i, \sigma_{i}>0$, and $[z]_{+}$denotes $\max (z, 0)$. In each sector we then determined the complementary GP distribution, conditional on exceeding the highest threshold, $u_{\max }=$ $\max \left(u_{i}: i=1, \ldots, k\right)$,

$$
\begin{aligned}
\operatorname{Pr}\left(X_{i}>\boldsymbol{x} \mid X_{i}>\right. & \left.u_{\max }\right) \\
& =\left[1+\frac{\xi_{i}\left(\boldsymbol{x}-u_{\max }\right)}{\tilde{\sigma}_{i}}\right]_{+}^{-1 / \xi_{i}}
\end{aligned}
$$

where $\tilde{\sigma}_{i}=\sigma_{i}+\xi_{i}\left(u_{\max }-u_{i}\right)$ is the 'modified' scale parameter (defined for $\tilde{\sigma}_{i}>0$ ). The omnidirectional distribution was then reconstructed as a weighted sum of complementary GP distributions in each sector [4]. Return periods, $\boldsymbol{N}$ (years) were calculated for a range of return levels $\boldsymbol{x}_{\boldsymbol{N}}$ from

$$
\begin{aligned}
& \frac{1}{\boldsymbol{N} n_{y}}=\sum_{i=1}^{k} \operatorname{Pr}\left(X_{i}>\boldsymbol{x}_{\boldsymbol{N}}\right) \\
& \quad=\sum_{i=1}^{k}\left\{\operatorname{Pr}\left(X_{i}>\boldsymbol{x}_{\boldsymbol{N}} \mid X_{i}>u_{\max }\right)\right. \\
& \left.\times \operatorname{Pr}\left(X_{i}>u_{\max } \mid X_{i}>u_{i}\right) \times \operatorname{Pr}\left(X_{i}>u_{i}\right)\right\} \\
& =\sum_{i=1}^{k}\left\{\left[1+\frac{\xi_{i}\left(\boldsymbol{x}_{\boldsymbol{N}}-u_{\max }\right)}{\tilde{\sigma}_{i}}\right]_{+}^{-\frac{1}{\xi_{i}}}\right. \\
& \left.\times\left[1+\frac{\xi_{i}\left(u_{\max }-u_{i}\right)}{\sigma_{i}}\right]_{+}^{-1 / \xi_{i}} \times \frac{n_{c, i}}{n}\right\}
\end{aligned}
$$

where $n_{c, i}$ was the total number of cluster peaks in the $i^{\text {th }}$ sector, and $n$ is the total number of samples. The resulting RP vs RL plot for the low-latitude magnetometer at Ascension Island $\left(\lambda=10.4^{\circ} \mathrm{S}\right)$ is presented in Figure 4, where the bold line (-) represents the MLE omnidirectional profile reconstructed from individual directional sector distributions (dot-dashed lines) (Eqn 4). The return levels are significantly smaller than those obtained by fitting to all data regardless of direction (- - -). Shading indicates the $95 \%$ CI determined by a bootstrapping method. This result is typical for sites below $40^{\circ}$ CGM latitude as illustrated by Figure 2, which compares the 100-year RLs using the directional sector reconstruction method (Eqn 8) ( $\square$ ) with those obtained ignoring direction $(+)$. Figure 2 also shows that reconstructing the RL from directional sectors often leads to higher RL value estimates at mid to high latitudes.

\section{Summary}

Applying extreme value theory to an extensive archive of magnetograms from 125 sites worldwide, we predict that return levels (RL) for $|d B / d t|$ peak sharply at $53^{\circ} \mathrm{CG}$ 
latitude (in both hemispheres) with a secondary peak near the geomagnetic poles at return periods of 100 years or more.

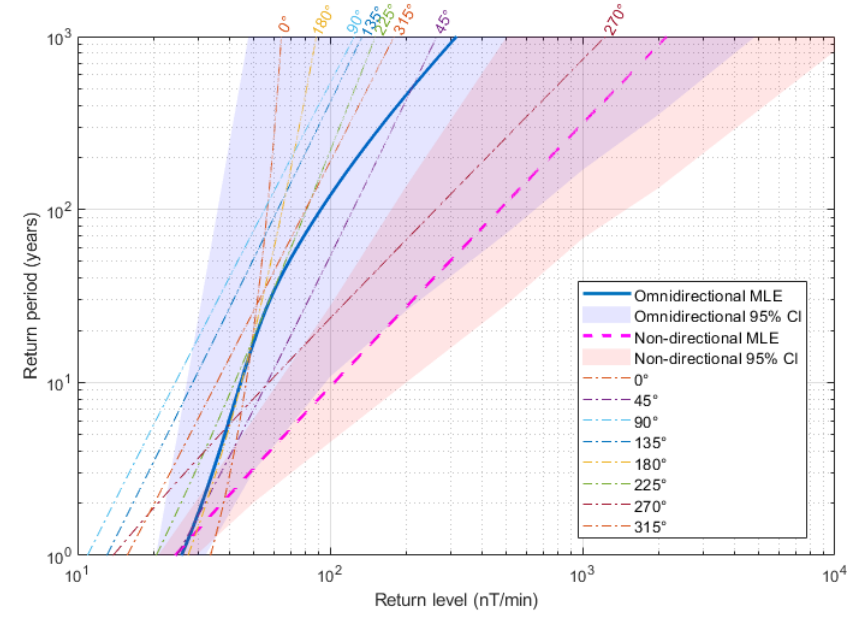

Figure 4. RP $v s$ RL at Ascension I. $\left(\lambda=10.4^{\circ} \mathrm{S}\right)$ for discretely sectored data $(\cdot-$ lines $)$. The solid blue line indicates the ML estimates reconstructed from discrete directional sectors (8). The magenta dashed line shows the ML estimates RLs ignoring directionality. Shading indicates 95\% Confidence Intervals.

Occurrence probabilities strongly depend on latitude, magnetic local time, month and direction, and are influenced by IMF orientation. Occurrence patterns match the known patterns of substorm expansions, Pc5 ULF waves, geomagnetic sudden commencements, and highlatitude lobe reconnection events. We have extended an earlier climatological study of 1-minute geomagnetic fluctuations [3] to cover both the ramp changes and RMS variations over periods from 1 to 60 minutes. The occurrence patterns depend strongly on the period of the disturbance and the direction of the field fluctuation. For example, large 60-minute ramp changes occur most frequently near noon at polar latitudes, whilst large 60minute RMS variations are most frequent in the auroral zone at 04-07 local time.

The weak assumption of identically distributed peaks is addressed by fitting GP distributions to data discretely sectored by direction (or month, MLT, etc.). Reconstructing distributions from directionally sectored data yields lower maximum likelihood estimates for RL for absolute geomagnetic latitudes below $40^{\circ}$ where there are strong directional anisotropies, but higher return levels above $40^{\circ}$. However the confidence intervals associated with this approach are much wider than those ignoring directionality.

\section{Acknowledgements}

This work was funded by the UK Natural Environment Research Council (NERC) under grant NE/P016715/1. Magnetometer data were provided by SuperMAG (available from http://supermag.jhuapl.edu) and we gratefully acknowledge contributions from the SuperMAG collaborators: Intermagnet; USGS, J. J. Love; CARISMA, PI I. Mann; CANMOS; The S-RAMP Database, PI K. Yumoto and Dr K. Shiokawa; The SPIDR database; AARI, PI Oleg Troshichev; The MACCS program, PI M. Engebretson, Geomagnetism Unit of the Geological Survey of Canada; GIMA; MEASURE, UCLA IGPP and Florida Institute of Technology; SAMBA, PI E. Zesta; 210 Chain, PI K. Yumoto; SAMNET, PI F. Honary; The IMAGE magnetometer network, PI L. Juusola; AUTUMN, PI M. Connors; DTU Space, PI A. Willer; South Pole and McMurdo Magnetometer, PI's L.J. Lanzarotti and A. T. Weatherwax; ICESTAR; RAPIDMAG; British Antarctic Survey; McMac, PI Dr P. Chi; BGS, PI Dr S. Macmillan; Pushkov Institute of Terrestrial Magnetism, Ionosphere and Radio Wave Propagation (IZMIRAN); GFZ, PI Dr J. Matzka; MFGI, PI B. Heilig; IGFPAS, PI J. Reda; University of L'Aquila, PI M. Vellante; BCMT, V. Lesur and A. Chambodut; Data obtained in cooperation with Geoscience Australia, PI M. Costelloe; AALPIP, co-PIs B. Clauer and M. Hartinger; SuperMAG, PI J. W. Gjerloev; Sodankylä Geophysical Observatory, PI T. Raita; Polar Geophysical Institute, A. Yahnin and Y. Sakharov; Geological Survey of Sweden, G. Schwartz; Swedish Institute of Space Physics, M. Yamauchi; UiT the Arctic University of Norway, M. G. Johnsen; Finnish Meteorological Institute, PI K. Kauristie.

\section{References}

1. L. Cagniard, 1953. "Basic theory of the magneto-telluric method of geophysical prospecting", Geophysics 18, 1953 pp. 605-635. doi: 606 10.1190/1.1437915.

2. I. A. Erinmez, J. G. Kappenman, and W. A. Radasky. 2002. "Management of the geomagnetically induced current risks on the National Grid 621 company's electric power transmission system", Journal of Atmospheric and Solar-Terrestrial Physics 64, March-April 2002, pp. 743756. doi: 10.1016/S1364-6826(02)00036-6.

3. N. C. Rogers, J. A. Wild, E. F. Eastoe, J. W. Gjerloev, and A. W. P. Thomson, "A global climatological model of extreme geomagnetic field fluctuations", Journal of Space Weather and Space Climate, 19 January 2020, doi: $10.1051 / \mathrm{swsc} / 2020008$

4. E. B. L. MacKay, P. G. Challenor, and A. S. Bahaj, "On the use of discrete seasonal and directional models for the estimation of extreme wave conditions", Ocean Engineering 37, April 2010, pp. 425-442. doi: 10.1016/j.oceaneng.2010.01.017.

5. J. W. Gjerloev, “A global ground-based magnetometer initiative", EOS 90, 3 June 2011, pp. 230-231. doi: 10.1029/2009EO270002.

6. S. Coles, An introduction to statistical modeling of extreme values. Springer-Verlag, London ltd.., 2001. 